\title{
Wegener's Granulomatosis Presenting as Bilateral Otalgia with Facial Palsy: A Case Report
}

\author{
Jeong Hyun Lee ${ }^{1}$, Kun Woo Kim ${ }^{1}$, Na Hye Myong ${ }^{2}$ and Jae Yun Jung ${ }^{1}$ \\ ${ }^{1}$ Departments of Otorhinolaryngology-Head and Neck Surgery, ${ }^{2}$ Pathology, Dankook University College of Medicine, Cheonan, Korea
}

Received March 18, 2013

Revised March 30, 2013

Accepted April 2, 2013

Address for correspondence

Jae Yun Jung, MD, PhD

Department of Otorhinolaryngology-

Head and Neck Surgery,

Dankook University

College of Medicine,

201 Manghyang-ro, Dongnam-gu,

Cheonan 330-715, Korea

Tel +82-41-550-6469

Fax +82-41-556-1090

E-mail jjkingy2k@gmail.com
Wegener's granulomatosis (WG) is defined as a granulomatous inflammation of the upper and lower respiratory tract due to a systemic vasculitis. Facial nerve palsy may occur during the course of any granulomatous or vasculitis disease. But very few cases have been reported in which facial nerve palsy is the presenting feature of WG. We recently experienced an interesting case of WG presenting as acute middle ear infection and subsequent bilateral facial palsy.

Korean J Audiol 2013;17:35-37

KEY WORDS: Earache · Facial paralysis · Wegener granulomatosis.

\section{Introduction}

Wegener's granulomatosis (WG) is a multisystem inflammatory disease. But, initial presentation may involve only head and neck symptoms. ${ }^{1,2)}$ These include nasal congestion, epistaxis, nose deformity, dyspnea, and alteration of the voice as well as symptoms of otitis media, mastoiditis, sensorineural hearing loss, and facial nerve palsy. ${ }^{3)}$ However, primary manifestation of WG in the mucosa of the middle ear is uncommon. ${ }^{3)}$ Furthermore WG presenting as synchronistic bilateral facial palsy is rare. The following case report presents our experience with WG patient whose main symptoms were middle ear inflammation and bilateral facial palsy. Bilateral facial palsy responded very well with medical treatment, but middle ear disease did not.

\section{Case Report}

A 59-year old woman visited our hospital for otalgia, hearing loss of both ears beginning a month ago. On examination, otoendoscopic examination revealed thickened and hyperemic tympanic membrane with granulation of the both ear (Fig. 1). In initial blood tests, serum white blood cells was within nor- mal limit (6470), erythrocyte sedimentation rate (ESR) and Creactive protein (CRP) were elevated $(33 \mathrm{~mm} / \mathrm{hr}, 1.10 \mathrm{mg} / \mathrm{dL})$ (Fig. 2). The rest of the routine hematological, biochemical tests and chest posteroanterior (PA) were normal.

The pure tone audiometry showed bilateral mixed hearing loss with bone conduction threshold $30-35 \mathrm{~dB}$. Computed tomography scanning of the temporal bone showed abnormal soft tissue densities in both mastoid, middle ear, epitympanum with left side worse. The canal of facial nerve was intact throughout its course.

Intravenous antibiotics treatment was started with impression of bilateral acute middle ear infection. On 6th day in hospital, we did myringotomy in left tympanic membrane and found yellowish granulation and scanty mucopus from middle ear. On 7th day, she expressed more otalgia and left facial nerve palsy (House-Brackmann grade III) was noticed. After considering aggravation of lest facial palsy ( $\mathrm{H}-\mathrm{B}$ grade $\mathrm{V})$, hearing deterioration, and continuous elevation of ESR and CRP, we decided to perform left mastoidectomy on 11th day (Fig. 2). Intraoperative finding showed loose granulation tissues filling entire mastoid and middle ear, which were easily sucked out with suction tip. Tissues were collected for bacterial, fungus, tuberculosis culture and pathologic evaluation. After left mas- 
toidectomy, her left hearing seemed to improve slightly because of reduced air-bone gap. But otalgia, facial expression, ESR and CRP didn't show any improvement. Eight days after left ear surgery, right otalgia was dramatic aggravated with development of right facial palsy (House-Brackmann grade V). Wide myringotomy was done. At this time point, pathologic report showed necrotizing granulomatosis with negative Acid Fast Bacillus, tuberculosis-polymerase chain reaction results (Fig. 3). We started high dose steroid treatment with suspicion of WG and checked serologic test including pancytoplastmic granular staining pattern anti-neutrophil cytoplasmic antibodies (c-ANCA) and rechecked chest PA. Follow-up chest PA showed enlarged both hilar mass lesions, which were not showed on initial chest PA (Fig. 4A, B). Subsequent chest CT showed multiple consolidations in both lungs with narrowing of left bronchus (Fig. 4C). Ophthalmological evaluation of her left eye revealed conjunctivitis and marginal keratitis (Fig. 4D).

Serology test showed elevated c-ANCA, antineutrophil antibodies, RA factor. Left facial expression started to recover. We transferred her to rheumatology department for further treatment. Her facial expression came back to normal function dur-

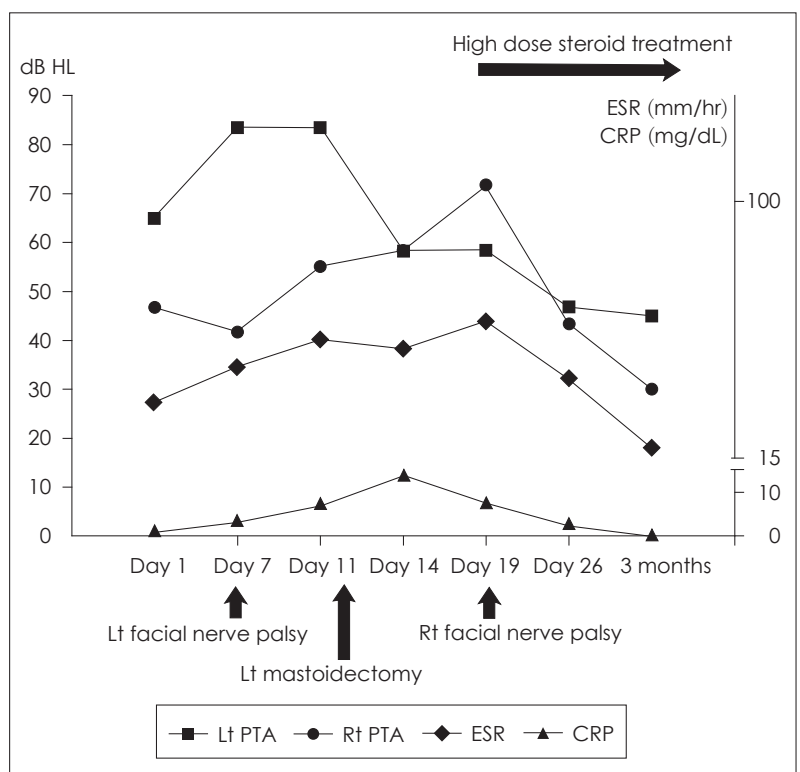

Fig. 2. Pure tone average, ESR, and CRP at different follow up time point $(1,7,11,14,19$, and 26 days and 3 months from first visit). The time points of surgical and medical intervention and facial palsy development were also indicated. PTA: pure tone average, ESR: erythrocyte sedimentation rate, CRP: C-reactive protein, $\mathrm{dB}$ HL: decibel hearing level.
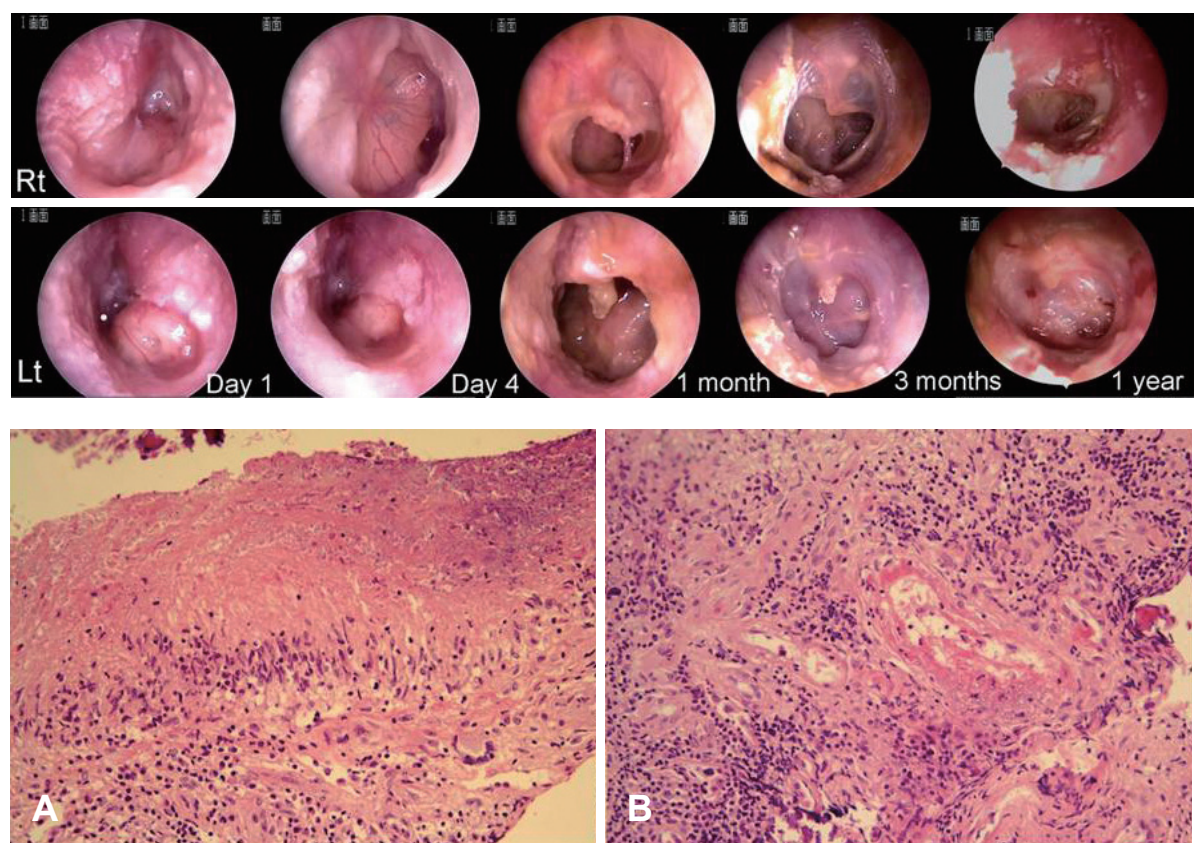

Fig. 3. A: Biopsy from left middle ear lesion revealed geographic necrotizing granuloma with rims of epithelioid histiocytes that are arranged in pallisading configuration and multinucleated giant cells. B: The necrotic lesions disclosed a necrotizing vasculitis that showed the homogenized eosinophilic necrosis of the walls and focally inflammatory cell infiltration (H\&E, $\times 400)$.
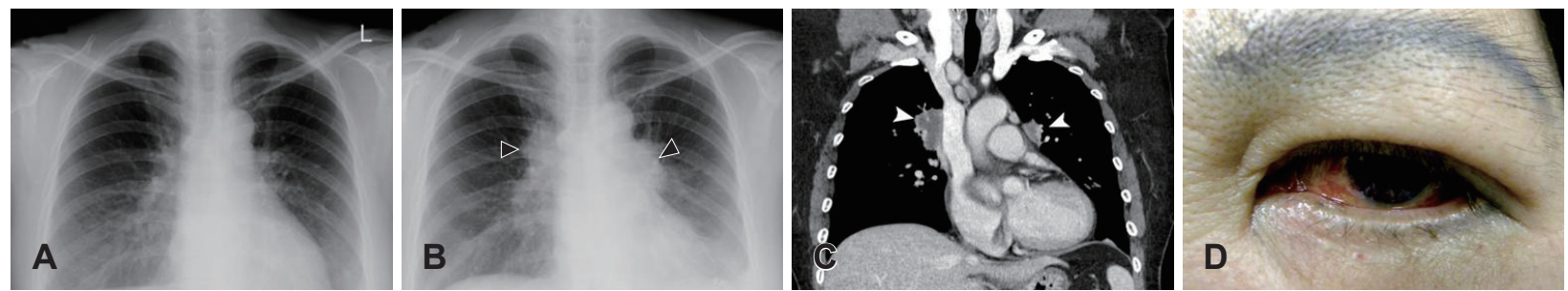

Fig. 4. A: Initial chest PA image showed no abnormal lesion. B: Follow-up chest PA revealed bilateral hilar enlargements (black arrowhead) and left pleural effusion. C: Chest CT scan showed multiple consolidations (white arrowhead) in both lungs. D: Patient also complaint left eye discomfort, which was diagnosed as marginal keratitis by ophthalmologist. Chest PA: chest posteroanterior. 
ing follow-up, but both tympanic membrane perforation and intermittent ear discharge and conductive hearing loss have remained until last follow-up (up to 1 year from first visit).

\section{Discussion}

WG is a disease that was first described by Friedrich Wegener in 1936 and is characterized histologically by necrotizing vasculitis of small arteries and veins. McCaffrey, et al. ${ }^{4)}$ reviewing 411 patients affected by the disease found that $72.3 \%$ presented head and neck involvement, while $71.2 \%$ had pulmonary symptoms and 57.5\% involvement of the kidneys. May and Klein ${ }^{5)}$ reviewing 2856 patients with facial palsy reported that only $2 \%$ presented with simultaneous bilateral involvement. Facial nerve palsy in WG is caused, as other cranial neuropathies, by compression in the temporal bone or vasculitis of the vasa nervosum. Nevertheless, a bilateral facial nerve palsy developing in the course of a bilateral otologic manifestation of WG has been reported extremely rarely in the literature. ${ }^{1)}$

In this case, left side facial nerve palsy developed earlier, and started to recover earlier than right side. Even though bilateral facial expression recovered fully, tympanic membrane perforation persisted and intermittent ear discharge continued to occur. Both side myringotomy (left wide myringotomy was done during mastoidectomy) might cause persistent tympanic membrane perforation later on. If diagnosis was made earlier before doing any surgical intervention, middle ear and tympanic membrane might have chance to heal more favorably.

The diagnosis of WG is made histologically by the presence of necrosis, granulomatous inflammation with multinucleated giant cells, vasculitis, and microabscess formation. A diagnosis of WG can be made on the basis of the clinical presentation, ANCA testing, and repeat biopsy specimens taken from the same or related sites. The specificity of positive c-ANCA testing in WG is greater than $95 \%$. ${ }^{6}$ The sensitivity of c-ANCA is only $65 \%$ to $70 \%$. C-ANCA test is highly sensitive for $\mathrm{WG}$, but a negative result does not exclude the diagnosis of $\mathrm{WG}$, it is clinically appropriate to interpret an increase in c-ANCA titer as an indicator to closely monitor the patient for signs of relapse.

The treatment of WG with cyclophosphamide and prednisone provides a $70 \%$ to $85 \%$ remission rate to an otherwise fatal disease. ${ }^{7)}$

\section{REFERENCES}

1) Nikolaou AC, Vlachtsis KC, Daniilidis MA, Petridis DG, Daniilidis IC. Wegener's granulomatosis presenting with bilateral facial nerve palsy. Eur Arch Otorhinolaryngol 2001;258:198-202.

2) Gubbels SP, Barkhuizen A, Hwang PH. Head and neck manifestations of Wegener's granulomatosis. Otolaryngol Clin North Am 2003; 36:685-705

3) Preuss SF, Stenner M, Beutner D, Laudes M, Klussmann JP. Fatal course of Wegener's granulomatosis with bilateral otomastoiditis and bilateral facial nerve palsy. Otolaryngol Head Neck Surg 2008;138: 799-800.

4) McCaffrey TV, McDonald TJ, Facer GW, DeRemee RA. Otologic manifestations of Wegener's granulomatosis. Otolaryngol Head Neck Surg (1979) 1980;88:586-93.

5) May M, Klein SR. Differential diagnosis of facial nerve palsy. Otolaryngol Clin North Am 1991;24:613-45.

6) Macias JD, Wackym PA, McCabe BF. Early diagnosis of otologic Wegener's granulomatosis using the serologic marker C-ANCA. Ann Otol Rhinol Laryngol 1993;102:337-41.

7) Illum $P$, Thorling K. Wegener's granulomatosis. Long-term results of treatment. Ann Otol Rhinol Laryngol 1981;90(3 Pt 1):231-5. 